Proceedings of the International Symposium on Physics of Materials (ISPMA 14), September 10-15, 2017, Prague

\title{
Comparison of Preferred Orientations of Zirconium-Alloy and Alpha Zirconium by Neutron and X-ray Diffraction
}

\author{
M. Kucerakova ${ }^{a} *$, S. Vratislav ${ }^{a}$, L. Kalvoda $^{a}$ And Z. Trojanova ${ }^{b}$ \\ ${ }^{a}$ Department of Solid State Engineering, Faculty of Nuclear Sciences and Physical Engineering, \\ Czech Technical University in Prague, Trojanova 13, 12000 Prague 2, Czech Republic \\ ${ }^{b}$ Department of Physics of Materials, Faculty of Mathematics and Physics, Charles University in Prague, \\ Ke Karlovu 5, 12116 Prague 2, Czech Republic \\ In our work, the textures of three zirconium-alloys tubes were compared with the textures of seven alpha \\ zirconium samples deformed by uniaxial tension from strain $5 \%$ to strain $30 \%$ (strain step 5\%). The texture \\ measurements by neutron diffraction were performed on the KSN-2 neutron diffractometer located at the re- \\ search reactor LVR-15 of the Research Centre Rež, Czech Republic. The X-ray measurements were performed at \\ theta/theta X'Pert PRO diffractometer with Cr X-ray tube. Observed data were processed by software packages \\ GSAS and X'Pert Texture. It was found that the (100) and (110) plane poles are oriented parallel towards the \\ rolling direction for both types of samples. All samples have preferred orientation of planes (102) and (103) per- \\ pendicular to normal direction. More significant tilt of basal poles from the normal direction toward the transverse \\ direction which indicates twinning was observed for alpha zirconium. Consequently, alloying of zirconium create \\ ideal texture with respect to zirconium hydride creation. The alloying of zirconium is reflected in decreasing the \\ overall texture sharpness. The obtained results are characteristic for zirconium.
}

DOI: 10.12693/APhysPolA.134.900

PACS/topics: 61.66.Bi; 61.66.Dk; 81.40.Ef; 61.05.cp; 61.05.fm

\section{Introduction}

The very low absorption cross-section of thermal neutrons, high hardness, ductility, and corrosion resistance of Zirconium makes it an attractive material for application in the nuclear industry as fuel rod cladding [1].

Most of the texture measurements are related to the basal pole distribution, which plays an important role. Less is known the distribution of prism or pyramidal plane poles. These are strong indicators for degree of annealing, and, moreover are important for quantitative determinations of textures, for example, by analysis of the orientation distribution function (ODF) $[2,3]$.

A very important issue is also the study of hydrides formation during service which has an impact on a decreased ductility and fracture toughness of the material [4].

In the alpha zirconium, slip takes place usually on the $\{10 \overline{1} 0\}$ first order prism planes along the $\langle 12 \overline{1} 0\rangle$ direction. Slip can occur also on the (0002) basal plane in the same direction. In regions of high stress concentration, the $\{10 \overline{1} 1\}$ slip is observed. Activation of slip with $\langle c\rangle$ component was noticed on first and second-order pyramidal planes $\{10 \overline{1} 1\}$ and $\{11 \overline{2} 1\}$ in $\langle c+a\rangle$ direction [2].

Most common twinning modes are $\{10 \overline{1} 2\}\langle\overline{1} 011\rangle$ tensile twins and $\{11 \overline{2} 2\}\langle\overline{1} \overline{1} 23\rangle$ compressive twins at room temperature and $\{10 \overline{1} 1\}\langle 10 \overline{1} 2\rangle$ twins at higher temperature [2].

\footnotetext{
* corresponding author; e-mail: monika.kucerakova@fjfi.cvut.cz
}

In this article, the preferred orientation of three zirconium-alloy tubes were confronted with preferred orientation of seven alpha zirconium samples. Samples were investigated by neutron and X-ray diffraction and preferred orientation parameters were determined by using pole figures and inverse pole figures.

\section{Samples}

\subsection{Alpha zirconium samples}

The alpha zirconium plate was forged and hot rolled to the thickness of $3.3 \mathrm{~mm}$. It was then annealed at $664^{\circ} \mathrm{C}$ and tensile specimens were produced from the plate. Figure 1 shows the shape, dimensions and coordination system of the samples (ND means Normal Direction, $\mathrm{RD}$ is Rolling Direction and TD is Transverse Direction). Table I shows the amount of the admixtures in the samples.

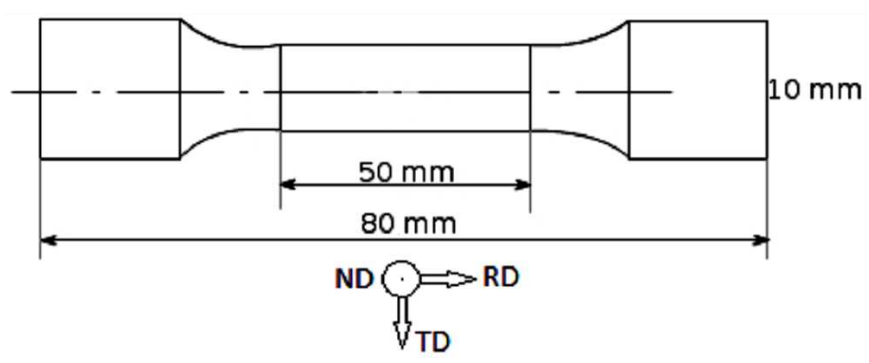

Fig. 1. Shape and dimensions of non-deformed alpha zirconium samples. 
TABLE I

Amount of admixtures in alpha zirconium samples

\begin{tabular}{c|c|c|c|c}
\hline \hline Hf [at.\%] & Ca [at.\%] & Mg [at.\%] & O [at.\%] & H [at.\%] \\
\hline 0.41 & 0.044 & 0.096 & 0.055 & 0.0011
\end{tabular}

The alpha zirconium samples were deformed on uniaxial tensile machine INSTRON 5882 from strain $5 \%$ to strain $30 \%$ (strain step was $5 \%$ ) at room temperature. The microstructure of the initial (non-deformed by uniaxial tension) sample observed by using light microscope Zeiss Axio Imager ZM1 is shown in Fig. 2. The grain size is about $20 \mu \mathrm{m}$.

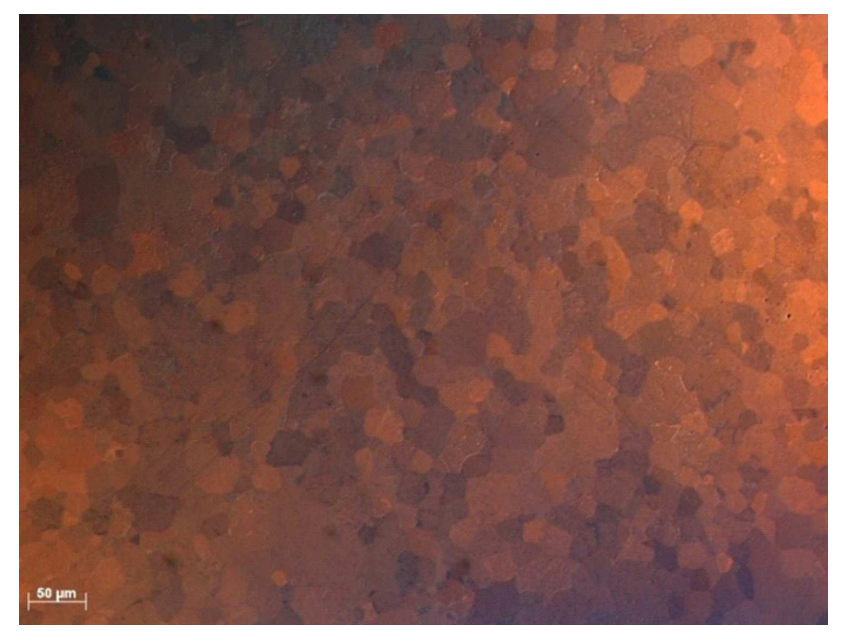

Fig. 2. Microstructure of non-deformed sample.

\subsection{Zirconium - alloys tubes}

All tubes (T1, T2 and T3) were extruded into an outside diameter of $50 \mathrm{~mm}$ and then cold-rolled with interannealing $\left(700^{\circ} \mathrm{C}\right)$ to the final sizes. Table II shows the composition and parameters of tubes $(v$ is tube length, $\varphi_{e}$ is outside tube diameter and $\varphi_{i}$ is internal tube diameter).

TABLE II

Chemical composition and parameters of tubes

\begin{tabular}{l|c|c|c}
\hline \hline & T1 & T2 & T3 \\
\hline Sn [wt.\%] & 1.46 & 1.39 & 1.42 \\
Fe [wt.\%] & 0.21 & 0.25 & 0.26 \\
Cr [wt.\%] & 0.09 & 0.11 & 0.12 \\
O [ppm] & 1090 & 1110 & 1080 \\
H [ppm] & 10 & 20 & 15 \\
N [ppm] & 50 & 60 & 45 \\
C [ppm] & 12 & 12 & 12 \\
$v$ [mm] & 39.6 & 30 & 30 \\
$\varphi_{e}[\mathrm{~mm}]$ & 12.1 & 9.1 & 9.2 \\
$\varphi_{i}[\mathrm{~mm}]$ & 11.2 & 8 & 8
\end{tabular}

\section{Diffraction experiments}

The neutronographic texture measurements were performed on the KSN-2 neutron diffractometer situated at the horizontal channel of the research reactor LVR-15 in the Nuclear Research Institute, plc. Rez, Czech Republic. The monochromatic neutrons having wavelength $0.1362 \mathrm{~nm}$ were used. The single-crystal $\mathrm{Cu}(200)$ was used as monochromator. The KSN-2 diffraction device offers good intensity and the best resolution value of $\Delta d / d=0.007$ in the region $d \sim 1.0 \div 0.1 \mathrm{~nm}(d$ is interplanar spacing). The samples were mounted in reflection geometry with its normal parallel to the direction of interest - usually RD, TD, or ND - and the reflected intensities were measured depending on Bragg angle. The resulting peak intensities were normalized to the intensities of a standard sample with random orientation of grains by using Mueller formula [5].

All the obtained neutronographic patterns were corrected for non-linear background and then evaluated using the Rietveld method implemented in the software package GSAS [6].

The X-ray texture measurements were performed at theta/theta X'Pert PRO diffractometer with Cr X-ray

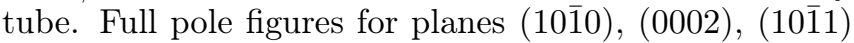
and $(11 \overline{2} 0)$ were calculated from incomplete pole figures using orientation distribution function (ODF). Observed data were processed by software package X'Pert Texture, PANalytical.

The intensity ratios $p_{h k l, q}$ calculated (for $q=\mathrm{ND}, \mathrm{RD}$ and TD) by using Mueller formula from neutronographic data are in Table III. Pole figures measured by X-ray

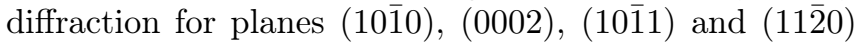
for alpha zirconium samples deformed at 5\%,15\% and $30 \%$ are given in Fig. 3. X-ray pole figures for tubes are in Fig. 4.

\section{Discussion}

Characteristic split of the basal pole maxima tilted from normal direction towards transverse direction can be observed for alpha zirconium samples (Fig. 3). This rotation implies twinning. Similar results can be seen from calculated intensity ratios for alpha zirconium in Table III. The value of pole density $p_{002, N D}$ for alpha zirconium does not significantly change with the increasing strain. On the other hand, the value of pole density $\mathrm{p}_{002, T D}$ shows a remarkable growth (from 1.27 to 3.30 , see Table III) during the deformation.

There is observed no tilt of basal poles for tubes in Xray pole figures (Fig. 4). However, a relatively high polar density in the transversal direction $p_{002, \mathrm{TD}}$ is visible for samples T2 $\left(p_{002, \mathrm{TD}}=1.39\right)$ and $\mathrm{T} 3\left(p_{002, \mathrm{TD}}=1.85\right)$.

It is evident from the X-ray pole figures that both alpha zirconium samples and tubes prefer orientation of planes $(10 \overline{1} 0)$ and $(11 \overline{2} 0)$ perpendicular to rolling direction (Figs. 3 and 4 ). The same conclusion can be seen from calculated pole density $\mathrm{p}_{100, R D}$ and $\mathrm{p}_{110, R D}$ (Table III). Moreover, for alpha zirconium it is obvious that the pole density $p_{100, \mathrm{RD}}$ slightly increases with the deformation (from 1.43 to 2.67) while the pole density $p_{110, \mathrm{RD}}$ slightly decrease with the increasing strain (from 1.72 to 0.87 ). 

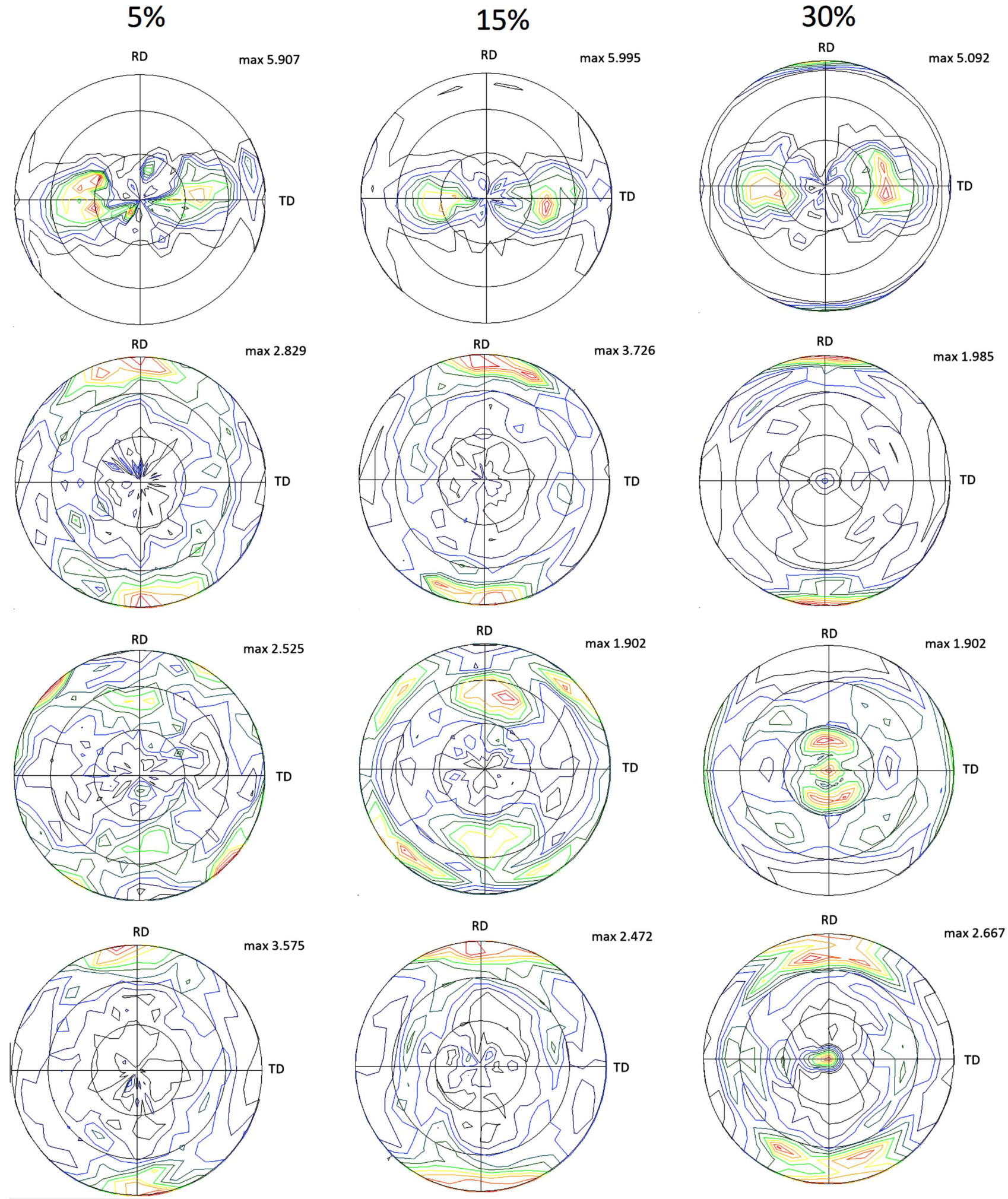

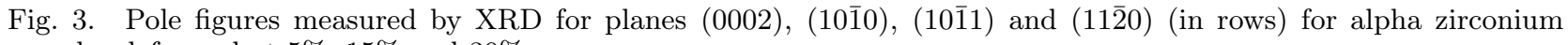
samples deformed at $5 \%, 15 \%$ and $30 \%$. 
T1

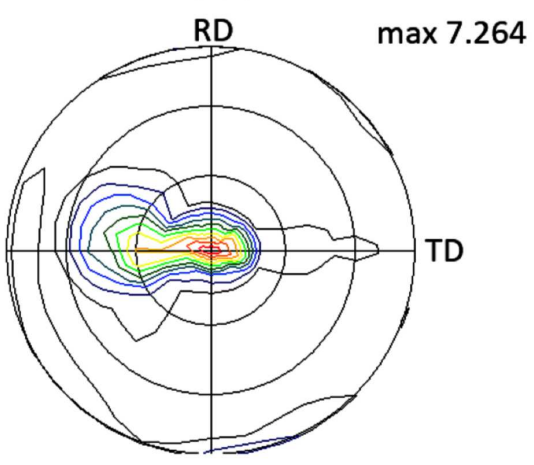

$\mathrm{RD}$
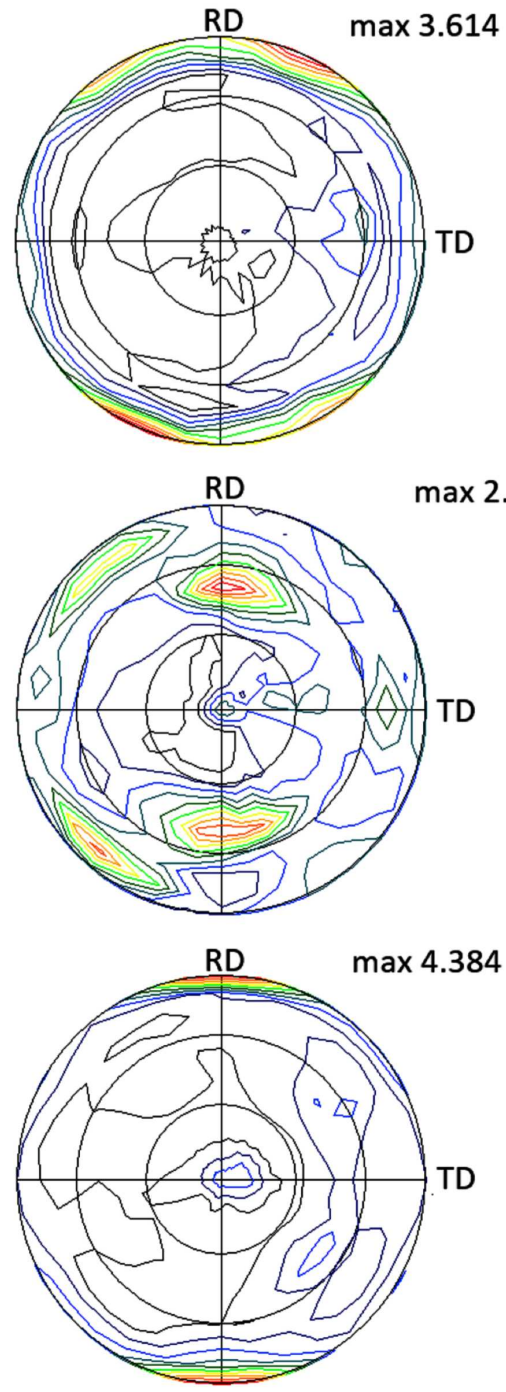

T2
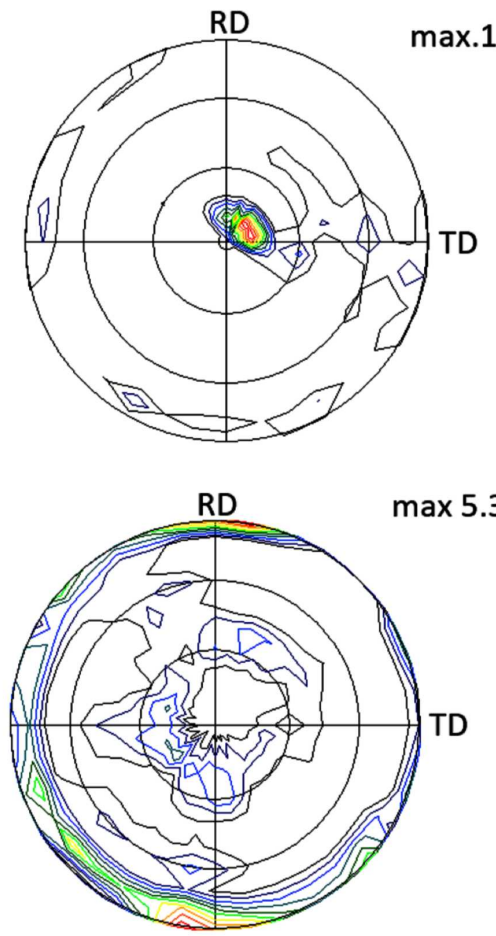

$\max 5.302$
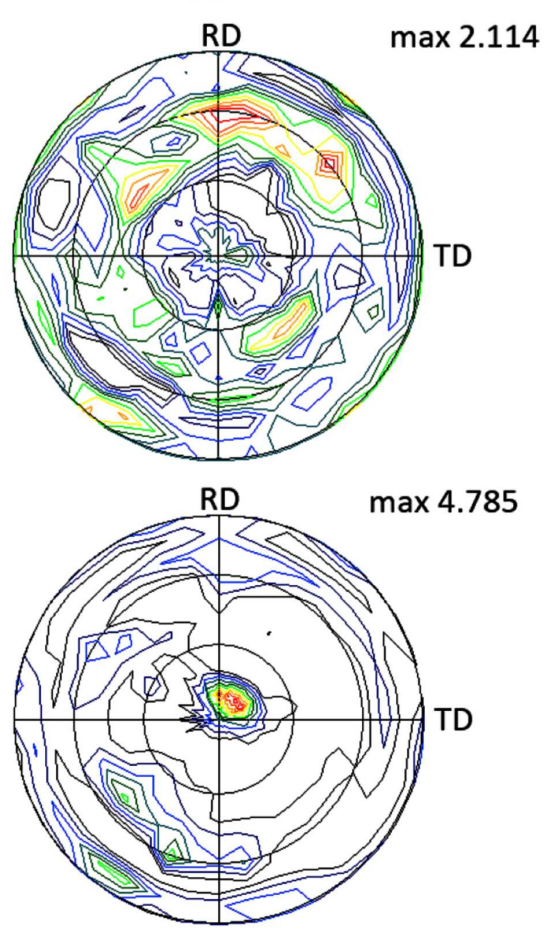

T3
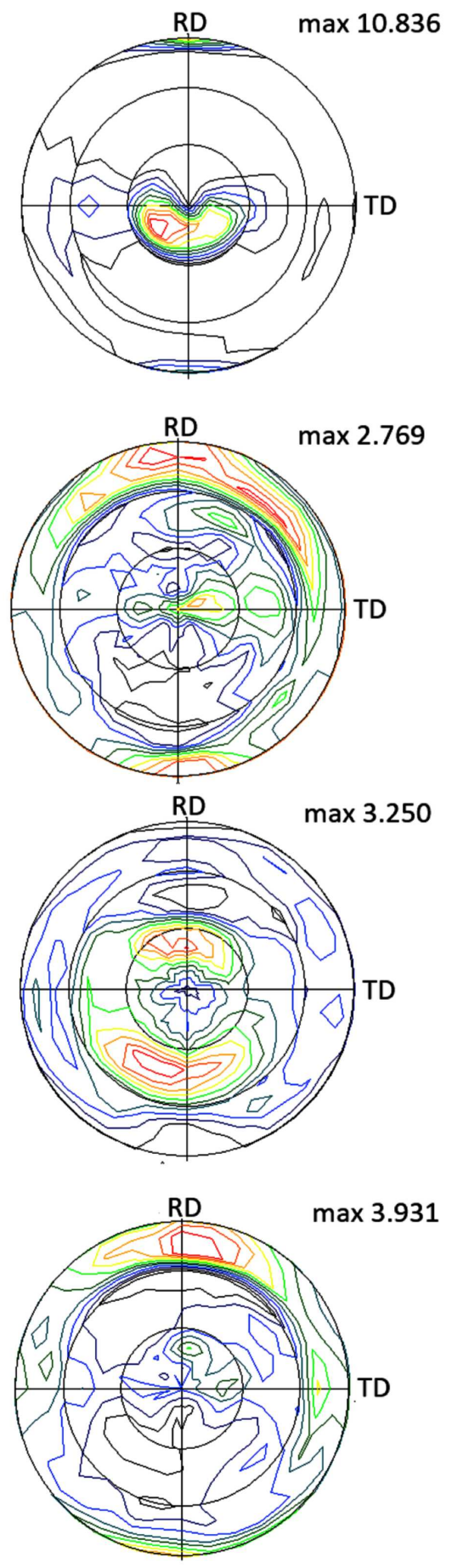

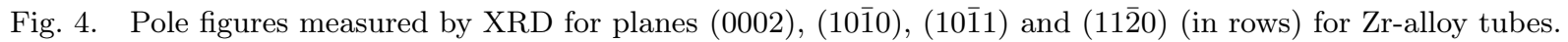

Samples prefer orientation of (1013) plane poles parallel to normal direction (Table III) and the value of pole density $p_{103, \mathrm{ND}}$ does not remarkably change during the deformation.

Planes (1012) also prefer orientation of their poles parallel to normal direction (see Table III), but for alpha zirconium samples, the pole density $p_{102, \mathrm{ND}}$ decreases with degree of deformation (from 1.74 to 0.47 ).

The apparent difference in results obtained from X-ray and neutron diffraction is probably due to the texture difference on the sample surface (investigated by X-ray diffraction) and in the volume (investigated by neutron diffraction) $[7,8]$. 
Inverse pole figures of alpha zirconium and zirconium tubes calculated from neutronographic measurements

\begin{tabular}{|c|c|c|c|c|c|c|c|}
\hline & $p_{002, \mathrm{ND}}$ & $p_{002, \mathrm{TD}}$ & $p_{100, \mathrm{RD}}$ & $p_{101, \mathrm{TD}}$ & $p_{110, \mathrm{RD}}$ & $p_{102, \mathrm{ND}}$ & $p_{103, \mathrm{ND}}$ \\
\hline $0 \%$ & 2.82 & 1.27 & 1.43 & 1.49 & 1.72 & 1.74 & 1.65 \\
\hline $5 \%$ & 2.67 & 1.73 & 1.51 & 1.10 & 1.45 & 1.58 & 1.41 \\
\hline $10 \%$ & 2.60 & 1.76 & 2.19 & 1.22 & 1.42 & 1.62 & 1.55 \\
\hline $15 \%$ & 2.77 & 2.01 & 2.49 & 1.01 & 1.45 & 1.36 & 1.34 \\
\hline $20 \%$ & 2.91 & 2.12 & 1.58 & 0.83 & 0.72 & 0.81 & 1.20 \\
\hline $25 \%$ & 2.85 & 2.27 & 2.34 & 1.31 & 0.83 & 0.34 & 1.52 \\
\hline $30 \%$ & 2.77 & 3.30 & 2.67 & 1.22 & 0.87 & 0.47 & 1.42 \\
\hline $\mathrm{T} 1$ & 1.70 & 0.62 & 1.17 & 0.69 & 2.78 & 1.10 & 1.80 \\
\hline $\mathrm{T} 2$ & 1.15 & 1.39 & 3.77 & 0.23 & 0.72 & 1.58 & 1.47 \\
\hline T3 & 1.17 & 1.85 & 2.95 & 0.14 & 0.79 & 0.59 & 1.13 \\
\hline
\end{tabular}

\section{Conclusions}

From the results reported above it can be seen that samples prefer orientation of poles of planes (1010) and $(11 \overline{2} 0)$ parallel to the rolling direction.

The split of basal poles toward the transverse direction indicate twinning. Thus, alloying of zirconium create ideal texture with respect to zirconium hydride creation [9].

Furthermore, samples prefer orientation of planes $(10 \overline{1} 2)$ and (1013) perpendicular to the normal direction. In alpha zirconium the level of resulting texture increases with deformation. The alloying of zirconium is reflected in increasing the overall texture sharpness. The obtained results are characteristic for zirconium $[10,11]$.

\section{Acknowledgments}

This work was supported by the Czech Science Foundation, grant No. GACR 14-36566G.

\section{References}

[1] K.L. Murty, in: Zirconium in the Nuclear Industry: Eighth International Symposium, Eds. L.F.P. Van Swam, C.M. Eucken, American Society for Testing and Materials, Philadelphia 1989, p. 570.

[2] E. Tenckhoff, J. ASTM Int. 2, 1 (2005).

[3] H. Hsun, Texture 1, 233 (1974).

[4] Z. Wang, et al., Metall. Mater. Trans. B 45, 532 (2013).

[5] G.B. Harris, Philos Mag. 43, 113 (1952).

[6] A.C. Larson, et al., General structure analysis system $(G S A S)$, Los Alamos National Laboratory Report LAUR 86-748, Los Alamos 2004.

[7] G.E. Bacon, Neutron Diffraction, Oxford, Clarendon Press, Oxford 1975, Ch. 2 and 3.

[8] H-R Wenk, et al., Rep. Prog. Phys. 67, 1367 (2004).

[9] I. Vetvicka, J. Nucl. Mater. 453, 196 (2014).

[10] V. M. Allen, et al., J. ASTM Int. 5, 1 (2008).

[11] J. R. Santisteban, et al., J. Nucl. Mater. 425, 218 (2012). 